\title{
Experimental Verification of Storm Sewer Transient Flow Simulation
}

\author{
Michał Szydłowski \\ Gdańsk University of Technology, Faculty of Civil and Environmental Engineering, \\ ul. G. Narutowicza 11/12, 80-233 Gdańsk, Poland, e-mail: mszyd@pg.gda.pl
}

(Received October 09, 2014; revised December 03, 2014)

\begin{abstract}
The paper focuses mainly on laboratory investigations of transient and transcritical flow in a single pipe of a sewer system. The aim of this paper is to present a comparison between pressure values calculated by an improved McCormack scheme and those measured at the hydraulic laboratory of the Gdańsk University of Technology, which were observed inside a pipe in an experiment for water flow with pressurization. The analysis proves that the numerical approach applied to flow simulation is a sufficiently accurate and reliable technique for predicting the basic parameters of storm sewer flow.
\end{abstract}

Key words: modeling, simulation, transient flow, storm sewers

\section{Introduction}

The process of rain water flow in systems of storm sewers is often affected by some characteristic hydraulic effects occurring in pipelines during high precipitation periods. When an extreme rainfall occurs, surface run-off from an urban basin can be relatively intensive, exceeding the capacity of sewer pipes. In such a situation the pipes may become completely filled with water, which results in a transition from free-surface to pressurized flow. After a storm event, the reverse transition can be observed. Such transitions are also possible in sewers when discharge is controlled by some control devices, such as gates, or as a result of pumps' operation. Moreover, rapidly varied flow with some local hydraulic effects, such as jumps or bores, can occur in water conduits during extreme storms.

Appropriate numerical methods have to be used to solve the storm sewer flow problem. In general, water flow in storm sewers can be classified as a free-surface open-channel flow because the pipes that transport water are partially filled and one-dimensional models are usually used to describe the flow. However, as already mentioned, hydraulic transitions and transcritical flow in sewer pipes can sometimes be observed. Many mathematical models of flow, adopting different techniques for 
transients implementation, can be found in the technical literature (Vasconcelos et al 2006). In general, the methods can be classified into three main groups, known as rigid column, full dynamic, and Preissmann slot models. In the Preissmann concept (Cunge et al 1980), a hypothetical slot is placed at the top of the pipe. The slot makes the flow free surface even when the pressure line in a pipe cross section is located above the top of the pipe. This method is very attractive for purposes of numerical calculation because there is no need to track the pressurization front. The Preissmann slot method can be found in many hydrodynamic models of storm sewer flow (Capart et al 1997, Trajkovic et al 1999), and it was also implemented and described by Szydłowski and Machalińska-Murawska (2012). The standard and improved McCormack schemes were used for the simulation of the transcritical flow by the latter authors. In order to assess the quality of the proposed numerical solution, results of simulations were compared with calculations and measurements available in the literature. It was concluded that an improved McCormack scheme both with idea of the Preissmann slot makes it possible to model transient and transcritical flow in storm sewer pipes, obtaining a fairly accurate description of the main flow features, such as positive and negative open-channel pressurized flow interfaces. However, owing to the unavailability of detailed information about experiment conditions and exact laboratory data to the authors of the above paper, the model was not conclusively verified. In order to asses the numerical solution more completely than merely on the qualitative level, precise measurements should be used for comparison. Now, the laboratory experiments have been carried out at the hydraulic laboratory of the Gdańsk University of Technology. The comparison between the author's numerical calculations and measurements of transient flow in a single storm sewer pipe is presented in this paper.

\section{Water Flow Model}

The free-surface flow in storm sewers can be described by the Saint-Venant equations (Cunge et al 1980), which are generally valid for gradually varied flow. During high surface water runoff from urban areas, various local phenomena, such as hydraulic jumps, can occur in sewers, and therefore a special form of flow model equations have to be used. To correctly reproduce steep water surface fronts, the conservative form of flow equations should be used in modeling (Cunge et al 1980). For the case of a prismatic channel, such as a pipe with no lateral inflow or outflow, the Saint-Venant system written in the conservative form can be presented as

$$
\frac{\partial \boldsymbol{U}}{\partial t}+\frac{\partial \boldsymbol{F}}{\partial x}=\boldsymbol{S}
$$

where the vectors $\boldsymbol{U}, \boldsymbol{F}$, and $\boldsymbol{S}$ are given as

$$
\boldsymbol{U}=\left(\begin{array}{l}
A \\
Q
\end{array}\right), \quad \boldsymbol{F}=\left(\begin{array}{l}
Q \\
\frac{Q^{2}}{A+I}
\end{array}\right), \quad \boldsymbol{S}=\left(\begin{array}{l}
0 \\
g A\left(S_{o}-S_{f}\right)
\end{array}\right),
$$


where $x$ represents distance along the sewer conduit, $t$ represents time, $A$ is a cross-sectional wetted area, $Q$ is flow discharge, $g$ is gravitational acceleration, and $I=p A / \rho$, where $p$ is a pressure at the centroid of $A$, and $\rho$ is a constant fluid density. For the hydrostatic pressure distribution, the term $I$ can be defined as $I=g A h c$, where $h c$ is a distance between the free surface and the centroid of the flow cross-sectional area. The $S_{0}$ and $S_{f}$ are the bottom and friction slopes, respectively. The friction slope can be defined by the Manning formula:

$$
S_{f}=\frac{n^{2} Q|Q|}{A^{2} R_{h}^{4 / 3}},
$$

where $n$ denotes the Manning friction coefficient, $R_{h}=A / P$ is the hydraulic radius, and $P$ is the wetted perimeter. Under free-surface flow conditions in a circular pipe of the inner diameter $d$, each geometrically related variable can be described by the wetted angle of the pipe $\theta$ (Fig. 1) (Leon et al 2009). The celerity $c$ is also related to the wetted angle $\theta$ because of the form of $A$ and the top width of the free surface $T$.

$$
c=\sqrt{\frac{g A}{T}} .
$$

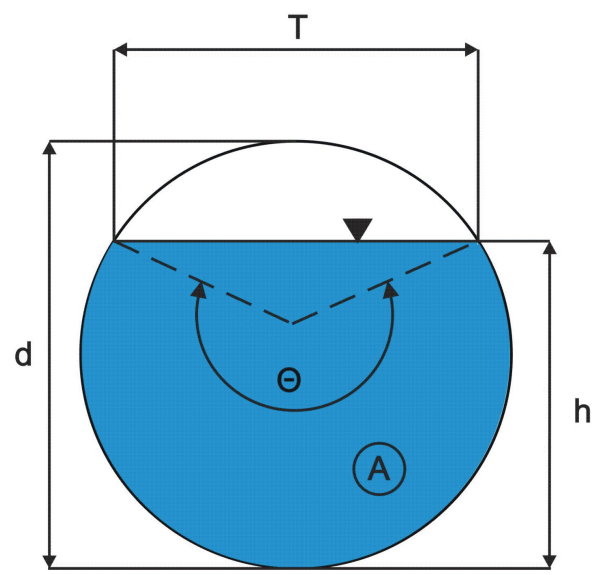

Fig. 1. Definition of variables in a circular cross section

In order to simulate water flow under pressurized conditions, the Preissmann slot concept is used in this work. The idea of a hypothetical slot at the top of the pipe was presented by Cunge et al (1980), and implemented for the numerical modeling of storm sewers, among others, by Capart et al (1997), Trajkovic et al (1999), and Leon et al (2009). It was also investigated by Szydłowski and Machalińska-Murawska (2012). The slot ensures that, after filling the pipe, the flow can be treated as the open-channel flow even if piezometric pressure exceeds the pipe diameter. In such a situation, the 
speed of a gravity wave in an open channel with a slot can be expressed according to the equation (4) as

$$
c=\sqrt{\frac{g A_{p}}{T_{s}}},
$$

where $A_{p}$ is the cross-sectional area of the pipe, and $T_{s}$ is the width of the slot. Moreover, the speed $c$ for the pressurized flow must be the same as the sound speed $a$ in a closed pipe.

The Preissmann slot idea makes the simulation of transient flow possible without the need to separately track the pressurization front, but this solution has some disadvantages. The most important is the inability to simulate subatmospheric full pipe flow, such as the one in some special segments of sewers of a siphon type. Other problems are inaccurate mass conservation, due to the additional slot in the pipe, and simulation instability when the water surface exceeds the pipe diameter and enters the slot.

The Saint-Venant equations $(1,2)$ are a system of partial differential equations, and their solution, for given initial and boundary conditions, is composed of functions $A(x, t)$ and $Q(x, t)$. In order to solve the model equations, a numerical method must be applied. In this paper, an improved McCormack scheme of the finite differences method (FDM) is used to solve the model equations. The improvement of the scheme is based on the theory of total variation diminishing schemes, which are capable of capturing sharp discontinuities without generating spurious oscillations. The scheme is second-order accuracy both in time and space in non-critical sections, but it switches the accuracy to the first-order at extreme points where the oscillations occur. This technique was previously described by Garcia-Navarro et al (1992), and it was originally proposed for solving one-dimensional open-channel flow equations. The scheme was used by Szydłowski and Machalińska-Murawska (2012) to simulate the transient flow in storm sewer pipe, and the results were compared with arbitrary data. In this paper, a previously developed numerical solution is evaluated against measurements carried out at the hydraulic laboratory of the Gdańsk University of Technology. This work is an element of a verification process of the hydraulic model of flow in a storm water pipeline.

\section{Laboratory Experiment}

The experimental set-up was built at the hydraulic laboratory of the Gdańsk University of Technology. The tests were carried out in a rectangular open channel with a concrete bed and glass walls, in which a circular pipe was located. The laboratory set-up featured a $10.55 \mathrm{~m}$ long pipe with a $0.15 \mathrm{~m}$ inner diameter. The Manning roughness coefficient was equal to $0.009 \mathrm{~s} / \mathrm{m}^{1 / 3}$. The pipe was constructed of two segments of the same bottom slope equal to 0.005 , and it joined two tanks. The water level in the downstream tank was initially kept below the pipe outlet. The upstream tank supplied 
the pipe with water. The experiment started from a steady state. In the inflow section, a constant discharge was kept. The scheme and photographs of the laboratory set-up are presented in Figs. 2 and 3.

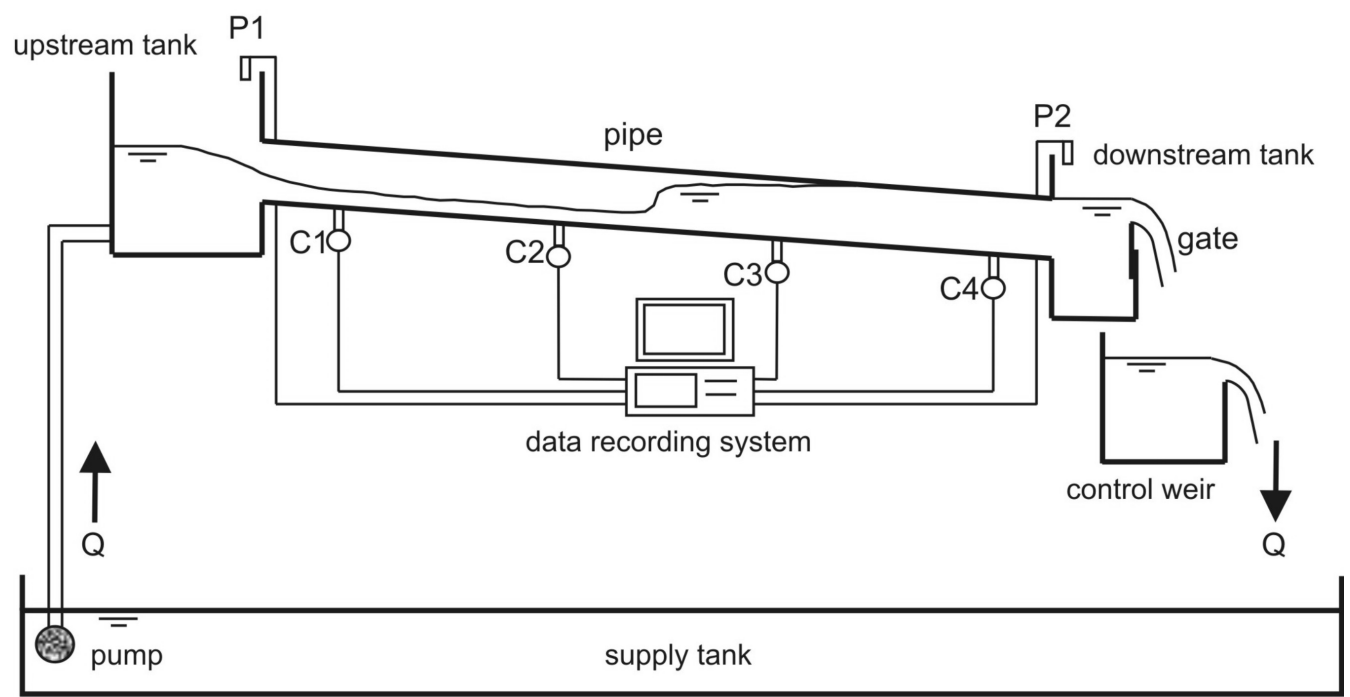

Fig. 2. Scheme of the laboratory set-up
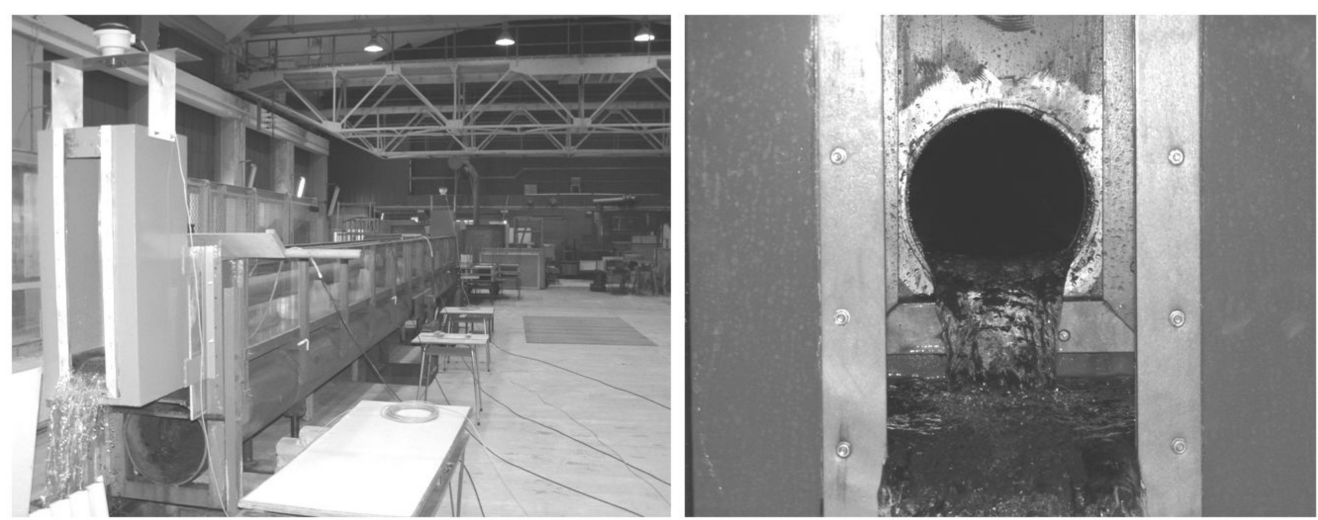

Fig. 3. Laboratory model of a single storm sewer pipe

The pressurization of pipe flow and the water surface front migrating upstream the pipe were generated by a sudden gate closure in the outflow section of the downstream tank. The variation of the pressure head in time was measured with hydrostatic probes at two points along the pipe located at $1.0 \mathrm{~m}(\mathrm{C} 4)$ and $6.54 \mathrm{~m}(\mathrm{C} 2)$ from the downstream end of the pipe. Additionally, water surface levels in both tanks were monitored by ultrasonic sounds (P1, P2). The locations of the other two probes (C3 
and C1), shown in Fig. 2, were not analyzed in this experiment because of a temporary failure of sensors.

The steady flow in the laboratory hydraulic system was measured with a control weir in the outlet section of the laboratory channel. The constant initial inflow rate was $0.0055 \mathrm{~m}^{3} / \mathrm{s}$. The water depth along the circular conduit was $0.062 \mathrm{~m}$. The unsteady flow and pipe pressurization were caused by a sudden maneuver in the outflow section. In the first phase of the experiment, after the sudden closure of the gate located in the downstream tank, a hydraulic jump was generated inside the pipe. Then the jump moved upstream, causing the pipe cross-section to fill completely with water. Finally, the pressurization process made the pipe function as a submerged culvert. In the final phase of the experiment, the gate in the downstream tank was opened in a short time, which led to a sudden decrease of the water surface. In this third phase, a fast transient in the form of a negative wave returned the flow to its initial conditions.

\section{Results and Discussion}

In order to simulate a one-dimensional water flow in a single pipe by the FDM according to the improved McCormack scheme, the conduit had to be transformed into a numerical mesh. The $10.55 \mathrm{~m}$ long pipe was discretized into 212 computational nodes with the spatial step $\Delta x=0.05 \mathrm{~m}$. The simulation was carried out with the time step $\Delta t=0.002 \mathrm{~s}$, which ensured the stability of the solution. The bottom line of the pipe was represented by a constant average value of the bottom slope in the simulation. The numerical simulation of transient flow was carried out for the same initial condition as described for the laboratory experiment. The hydraulic jump, which started the pressurization process, was generated in the outflow section of the pipe by a sudden closure of the gate in the downstream tank. The variation of the water level measured in the tank, presented in Fig. 4, was used as the downstream boundary condition for the flow simulation. It can be observed that the pressurization of the outflow section of the pipe began at time $t=14.5 \mathrm{~s}$ and finished at $t=70 \mathrm{~s}$.

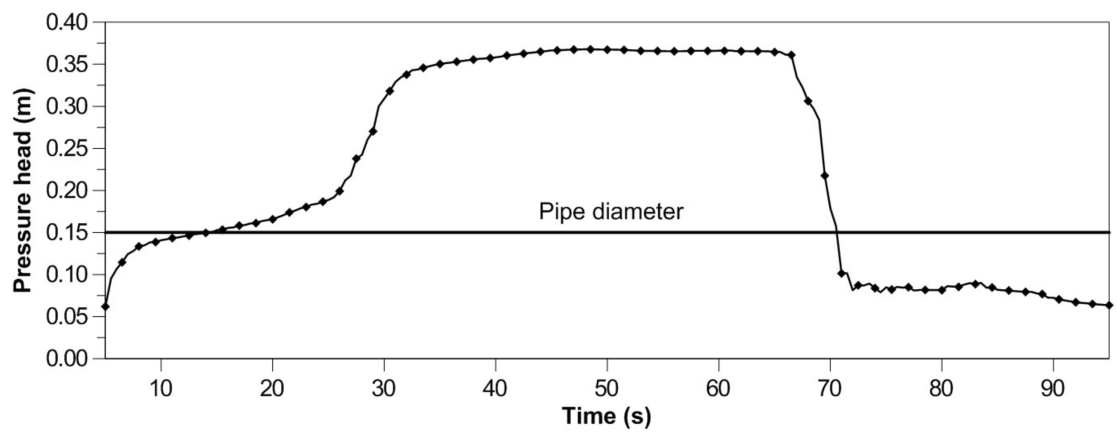

Fig. 4. Downstream boundary condition - pressure measured at control point P2 


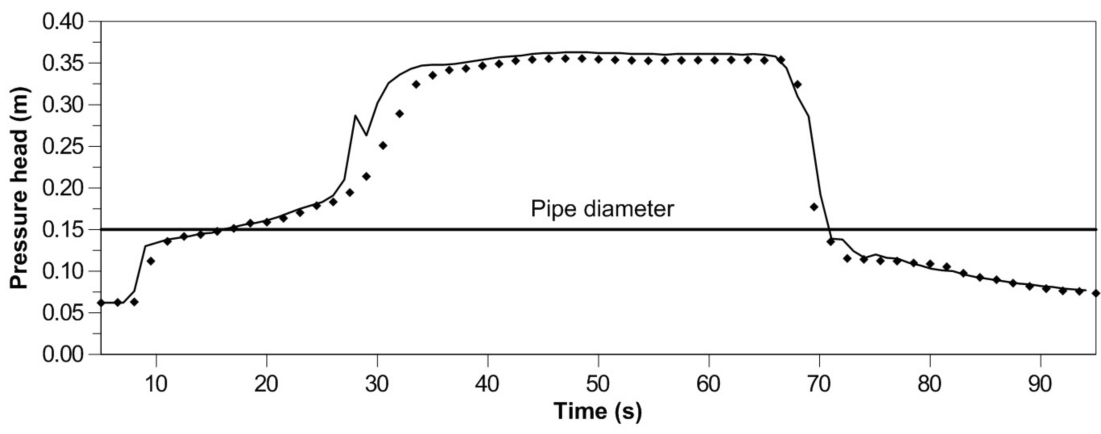

Fig. 5. Measured (markers) and computed (line) pressure at control point C4

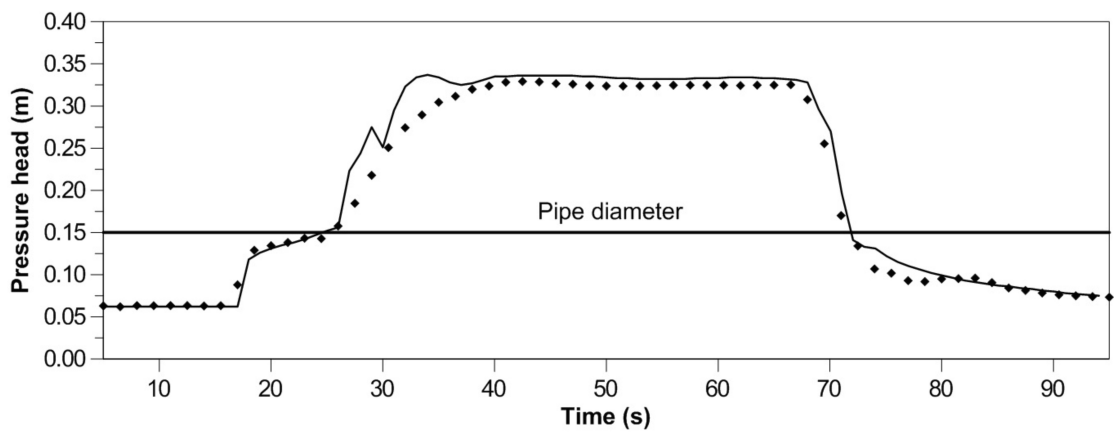

Fig. 6. Measured (markers) and computed (line) pressure at control point C2

The comparison between measurements and the results of numerical simulation is presented in Figs. 5-11. The graphs in Figs. 5, 6, and 7 show the variation in time of the pressure head for three measurement points $\mathrm{C} 4, \mathrm{C} 2$ and $\mathrm{P} 1$, respectively. It can be observed that just after the gate closure, a hydraulic jump was formed inside the conduit, which can be seen as a sudden rise of the water depth in the pipe. The hydraulic jump migrated upstream the pipe under free-surface flow conditions, reaching the control points $\mathrm{C} 4$ and $\mathrm{C} 2$ at $t=9 \mathrm{~s}$ and $t=16 \mathrm{~s}$, respectively. After $t=25.5 \mathrm{~s}$, the hydraulic jump reached the upstream tank (point P1), and started to fill it with water (Fig. 7). Owing to the continuously rising water level in the downstream tank, the pressurization of the pipe began after the hydraulic jump. This effect can be observed at points $\mathrm{C} 4$ and $\mathrm{C} 2$ at times $t=17 \mathrm{~s}$ and $t=25 \mathrm{~s}$, respectively, when the pressure head exceeds the pipe diameter. Both these hydraulic phenomena, related to rapidly varied and transient water flow, were reproduced quite accurately in the numerical simulation. The discrepancy between measurements and computations observed at the beginning of the pressure pipe flow period results from the oscillation of the numerical solution due to the application of the Preissmann slot technique. 


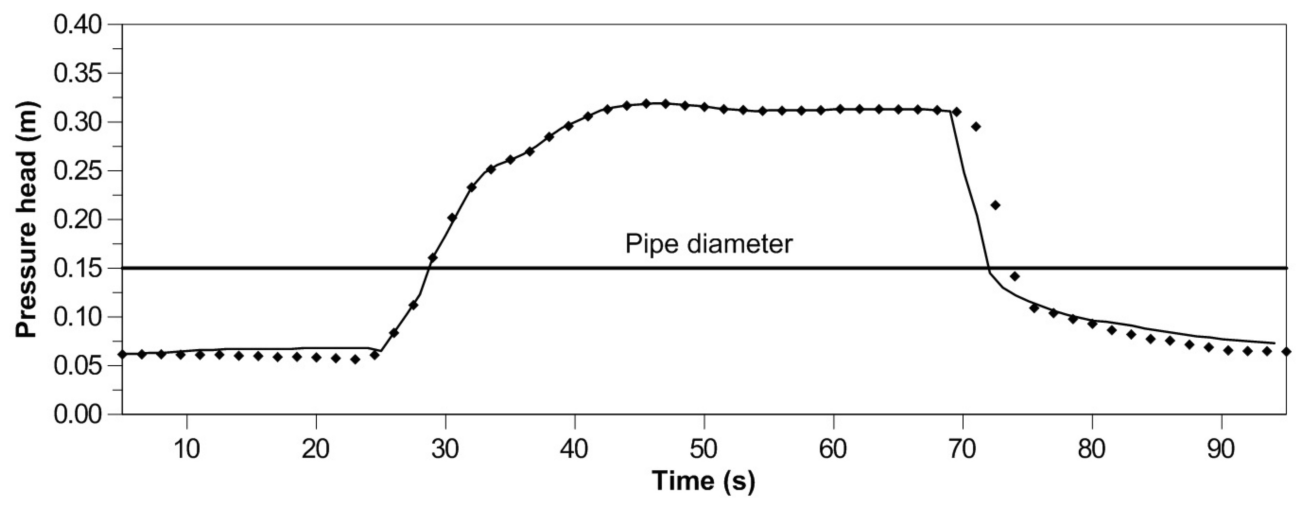

Fig. 7. Measured (markers) and computed (line) pressure at control point P1

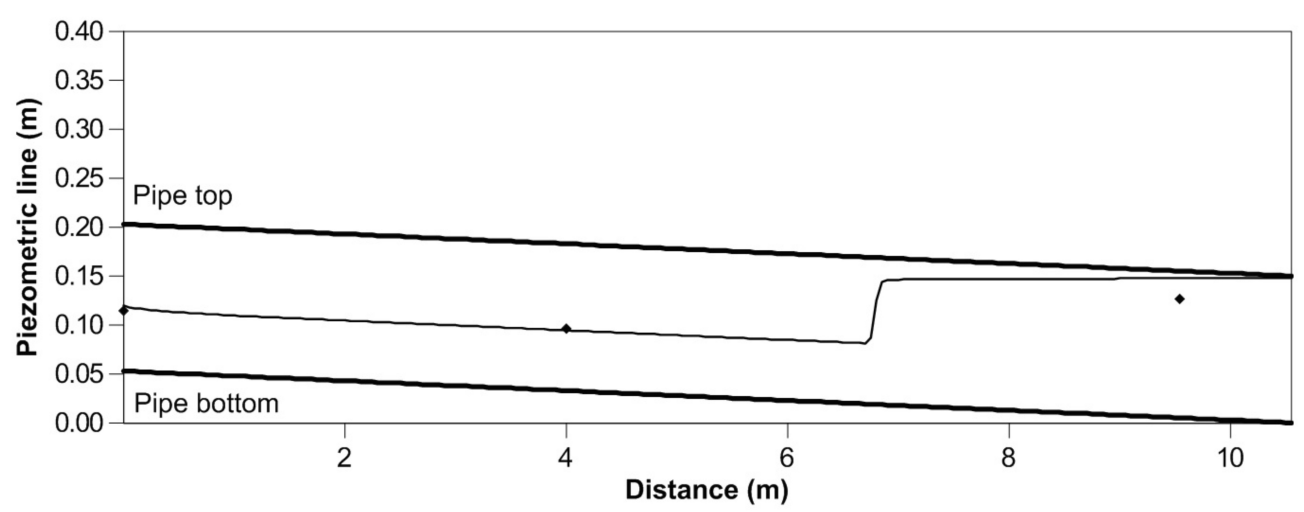

Fig. 8. Measured (markers) and computed (line) pressure profiles for $t=10 \mathrm{~s}$

After the full pressurization of the pipe, the water flow became steady. During this period, the piezometric pressure along the pipe was almost the same because of the small flow velocity and the low value of the pipe friction coefficient. The flow became unsteady again at $t=69 \mathrm{~s}$ because of the opening of the gate in the downstream tank. The sudden decrease in pressure and the transition from pressure flow to free-surface flow can also be observed for control points C4, C2 and P1 (Figs. 5, 6, 7). First, it can be seen in the $\mathrm{C} 4$ section, where pressurization finished at $t=70 \mathrm{~s}$. Next, the free-surface flow occurred at point $\mathrm{C} 2$ at $t=72 \mathrm{~s}$, and finally in section P1 at $t=74$ s. The worst quality of the numerical simulation was obtained for point P1, where the inflow boundary condition was not precisely imposed for the calculation.

The processes of hydraulic jump migration and pressurization of the pipe can also be observed in Figs. 8, 9, 10, and 11, which compare computed and measured pressure profiles for selected points in time. In Figs. 8 and 9 the hydraulic jump moving upstream can be seen at $t=10 \mathrm{~s}$ and $t=20 \mathrm{~s}$, respectively. It can also be noted that 


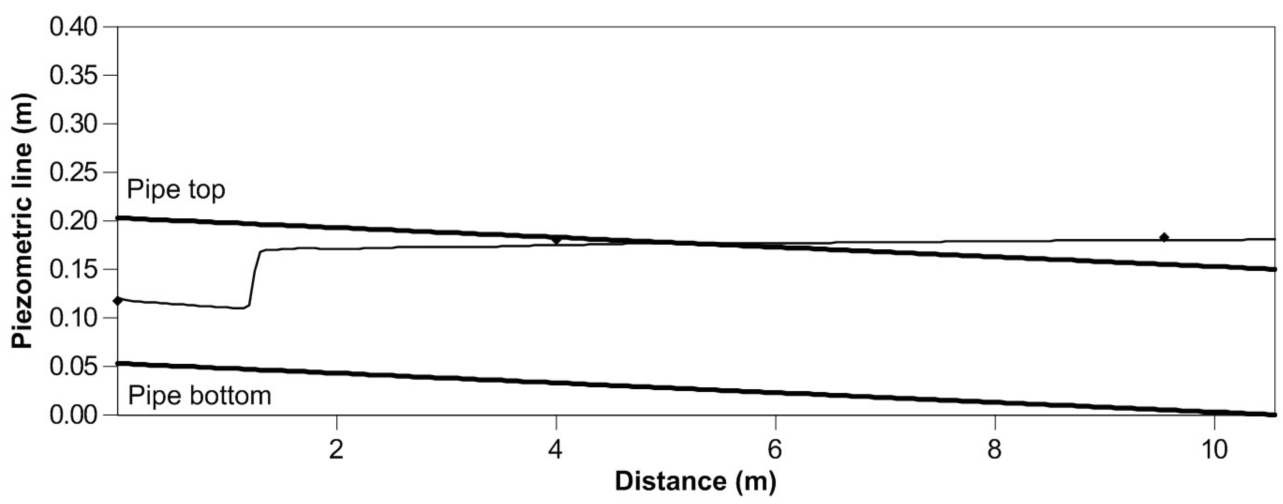

Fig. 9. Measured (markers) and computed (line) pressure profiles for $t=20 \mathrm{~s}$

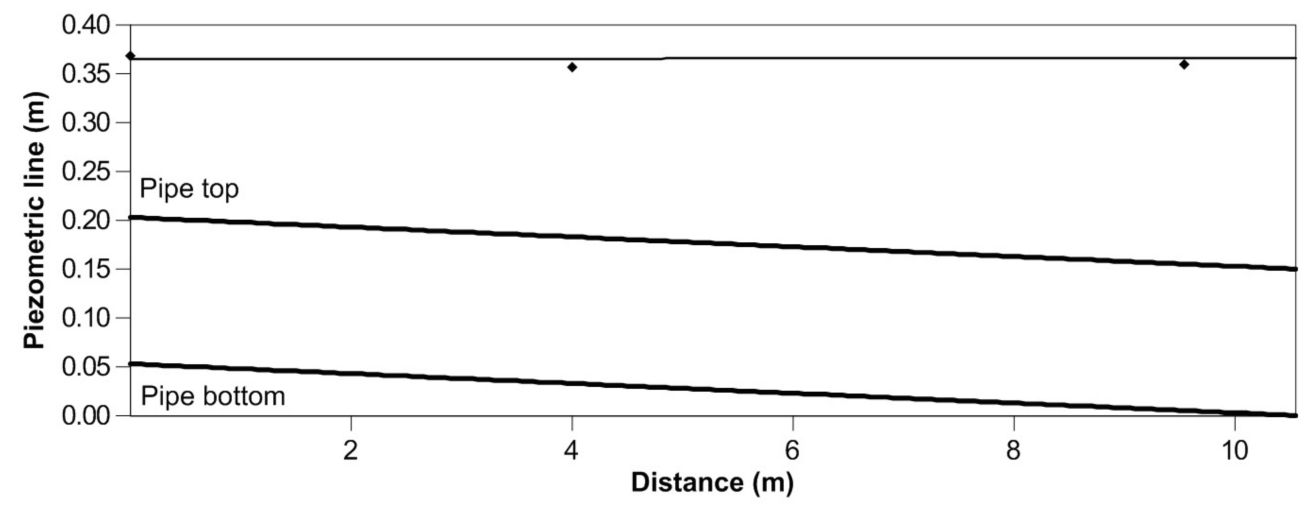

Fig. 10. Measured (markers) and computed (line) pressure profiles for $t=50 \mathrm{~s}$

after 20 seconds of simulation, the upper part of the pipe is partially filled with water, and the lower part is pressurized. Fig. 10 represents the steady flow state under submerged conditions observed 50 seconds after the gate closure. Finally, the pressure profile in the form of free water surface for $t=75 \mathrm{~s}$ is presented in Fig. 11. Satisfactory agreement between calculations and measurements was obtained for most test cases, which proves that the solution of unsteady water flow equations for transient flow by the improved McCormack scheme was appropriate. Disagreement can be observed only after the sudden release of water (Fig. 11), which can be explained by the poor representation of the outflow boundary condition during this experiment period. 


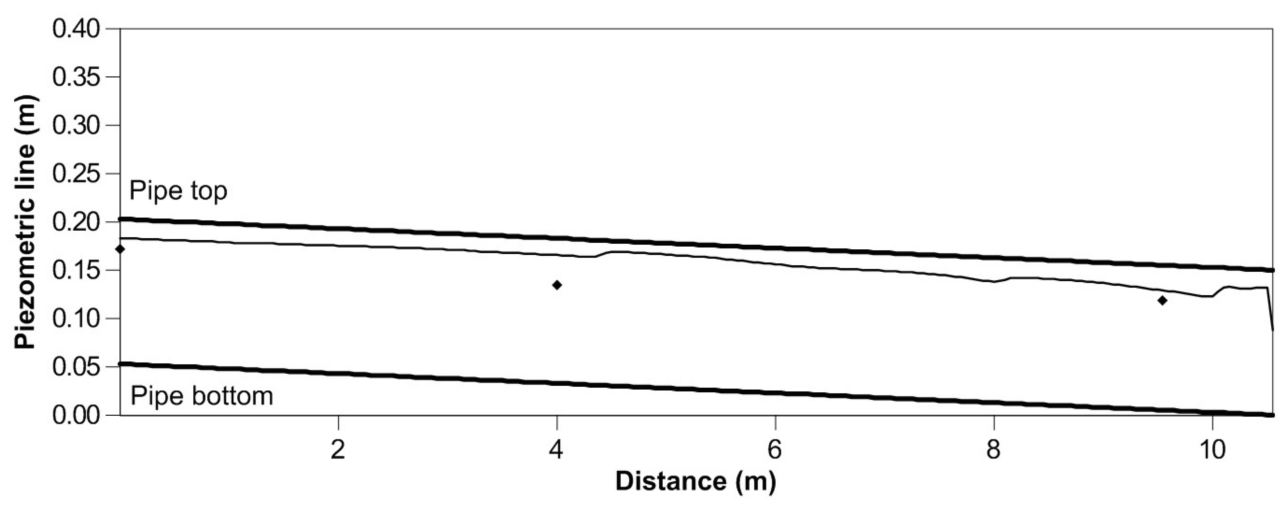

Fig. 11. Measured (markers) and computed (line) pressure profiles for $t=75 \mathrm{~s}$

\section{Conclusion}

The main aim of the research was to verify experimentally the choice of Saint-Venant equations as a hydraulic model of transient and transcritical flow simulation in storm sewers. Additionally, an applied numerical method for the solution of model equations by an improved McCormack scheme and the Preissmann slot technique for pressurized flows was examined. The results of numerical simulations of flow in a single conduit were presented and analyzed in the paper. They were compared with measurements carried out by the author in a hydraulic laboratory. It was ascertained that hydraulic phenomena characteristic of storm sewers were accurately simulated. The experiment presented in this paper makes it possible to find a satisfactory numerical solution of Saint-Venant equations for the modeling of drainage systems. It seems that the solution technique applied can be useful for future work on dual drainage modeling (storm sewers - surface), offering a more realistic representation of urban flooding processes.

\section{Acknowledgments}

Author expresses gratitude to the Polish National Science Center for the research project N N523 745840.

\section{References}

Cunge J. A., Holly F. M. and Verwey A. (1980) Practical Aspects of Computational River Hydraulics, London, Pitman Publ. Ltd.

Capart H., Sillen X. and Zech Y. (1997) Numerical and Experimental Water Transients in Sewer Pipes, J. Hydraul. Res., 35, 659-672.

Garcia-Navarro P., Alcrudo F. and Saviron J. M. (1992) 1-D Open-Channel Flow Simulation Using TVD-McCormack Scheme, J. Hydraul. Eng., 118, 1359-1372. 
Leon A. S., Ghidaoui M. S., Schmidt A. R. and Garcia M. H. (2009). Application of Godunov-type Schemes to Transient Mixed Flows, J. Hydraul. Res., 47, 147-156.

Szydłowski M. and Machalińska-Murawska J. (2012) Numerical Simulation of Transient Flow in Storm Sewers Using Standard and Improved McCormack Scheme, Task Quarterly, 16, 53-74.

Trajkovic B., Ivetic M., Calomino F. and D'Ippolito A. (1999) Investigation of Transition from Free Surface to Pressurized flow in a Circular Pipe, Wat. Sci. Tech., 39, 105-112.

Vasconcelos J. G., Wright S. J. and Roe P. L. (2006) Improved Simulation of Flow Regime Transition in Sewers: Two-Component Pressure Approach, J. Hydraul. Eng., 132, 553-562. 